\title{
ON THE RELATION OF CHANGES IN BLOOD VELOCITY AND VOLUME FLOW OF BLOOD TO CHANGE OF POSTURE
}

\author{
By A. V. BOCK, D. B. DILL AND H. T. EDWARDS \\ (From the Fatigue Laboratory, Morgan Hall, Harvard University, and the Medical Department \\ of the Massachusetts General Hospital, Boston, Massachusetts)
}

(Received for publication November 20,1929)

Recently Weiss, Robb, and Blumgart (1) measured the velocity of blood flow in a large series of normal and diseased subjects by means of suitable doses of histamine phosphate injected intravenously. All subjects were studied in the prone position. The results obtained were checked in ten normal persons by simultaneous measurements of the velocity of blood flow by the radioactive deposit method (2). The latter observations showed that the reaction time of histamine was about 5 seconds slower than the average arm to arm circulation time estimated by the radium deposit method, and that the differences were relatively constant. The evidence in general presented by this work indicates that the use of histamine provides a simple method of measuring the least circulation time in man.

The present work was undertaken to ascertain what differences in the reaction time of histamine may exist when the posture of the subject is changed. If the volume flow of blood remains constant in both the lying and standing positions, the inference follows that the velocity of blood flow would probably remain about the same. Under such conditions the reaction time of histamine would be in close agreement for both positions. A change of volume flow of blood, on the other hand, with change of posture, would be suggested if the velocity of the histamine reaction time differed greatly in the two positions.

\section{EXPERIMENTAL}

The subjects used in this work are all men in normal health, engaged for the most part in laboratory work. Each subject rested flat on the bed in a non-fasting state for at least 15 minutes, or stood still not less 


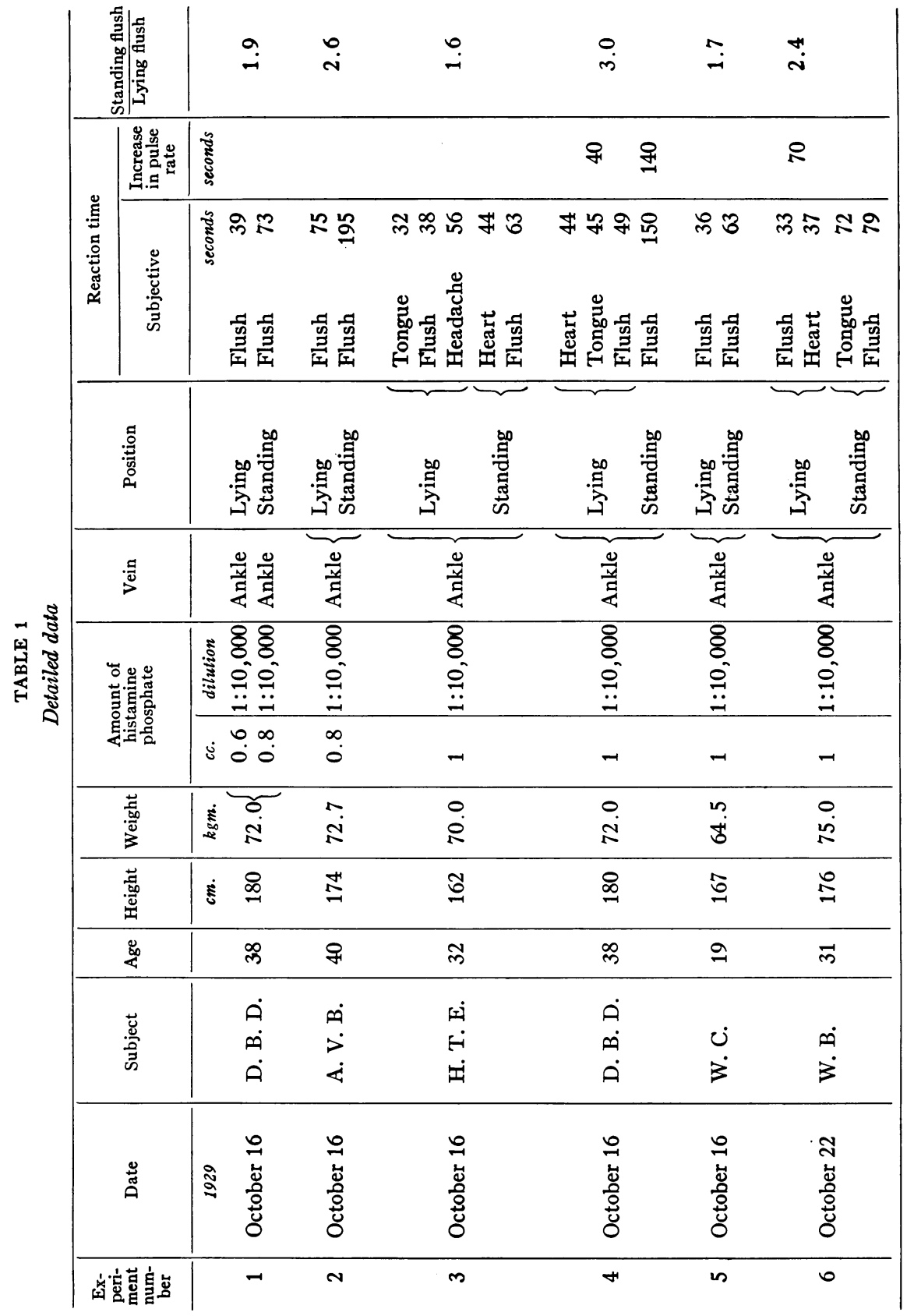




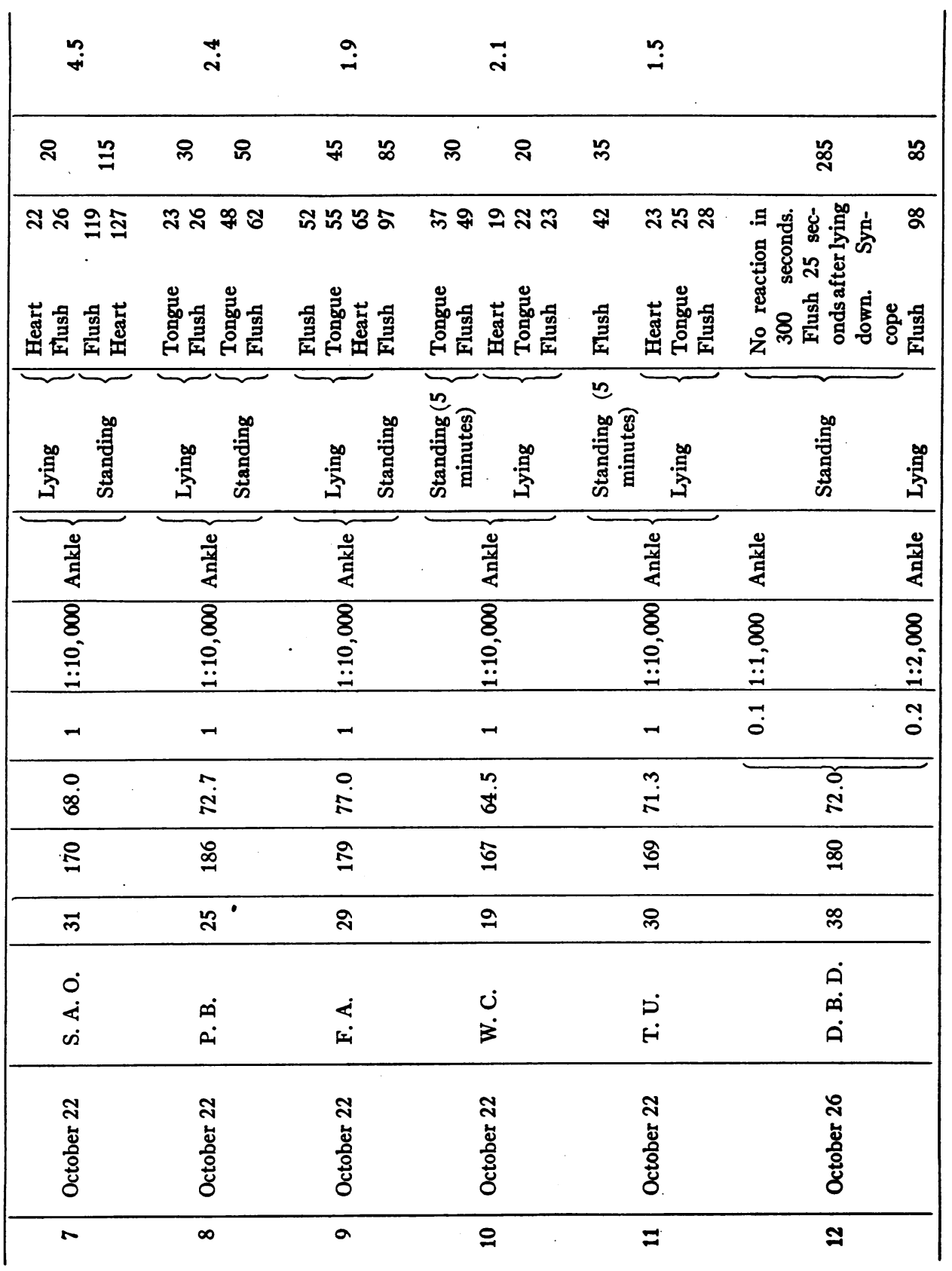

THE JOURNAL OF CLINICAL INVESTIGation, vOL. viI, No. 4 


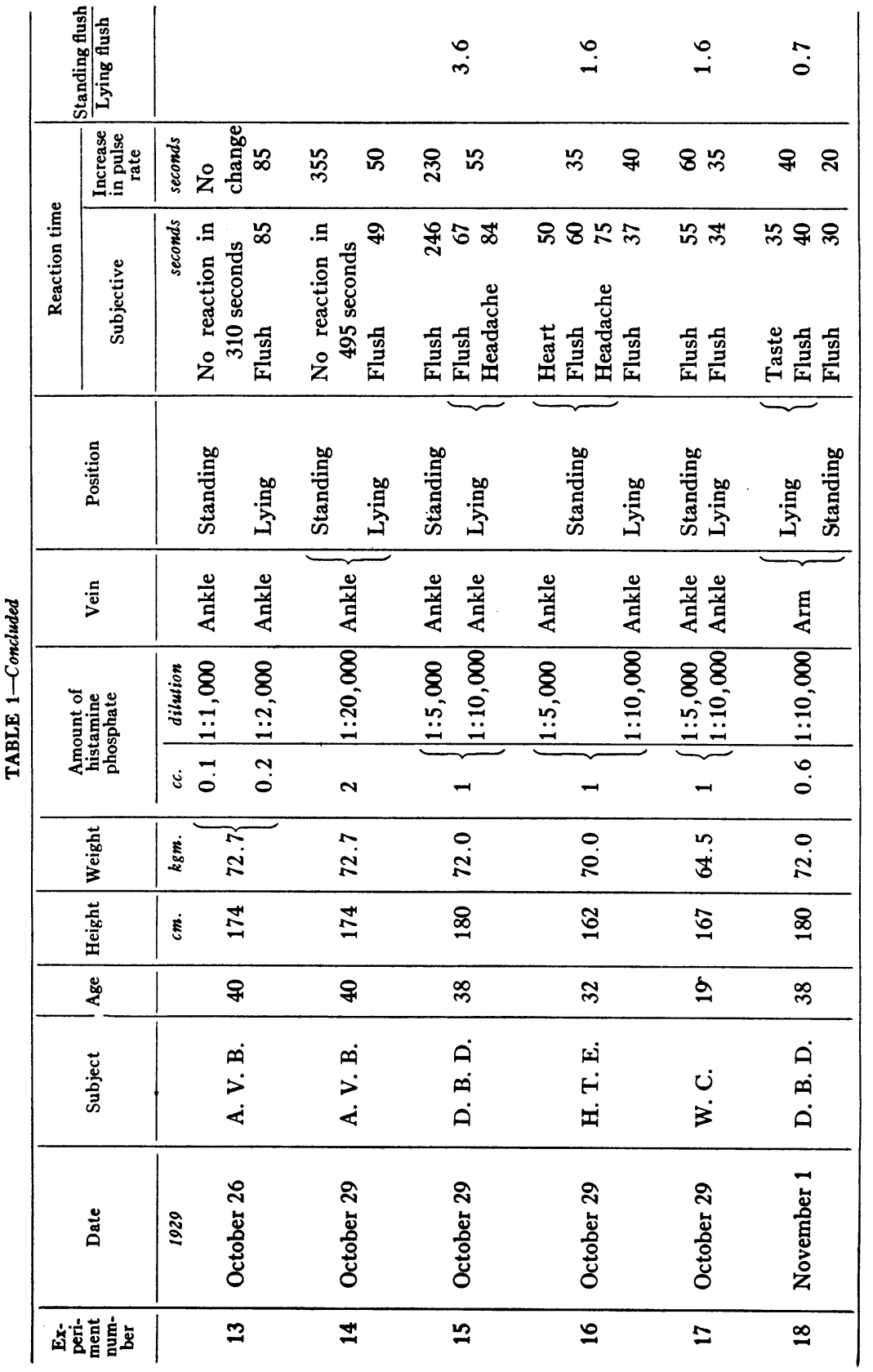




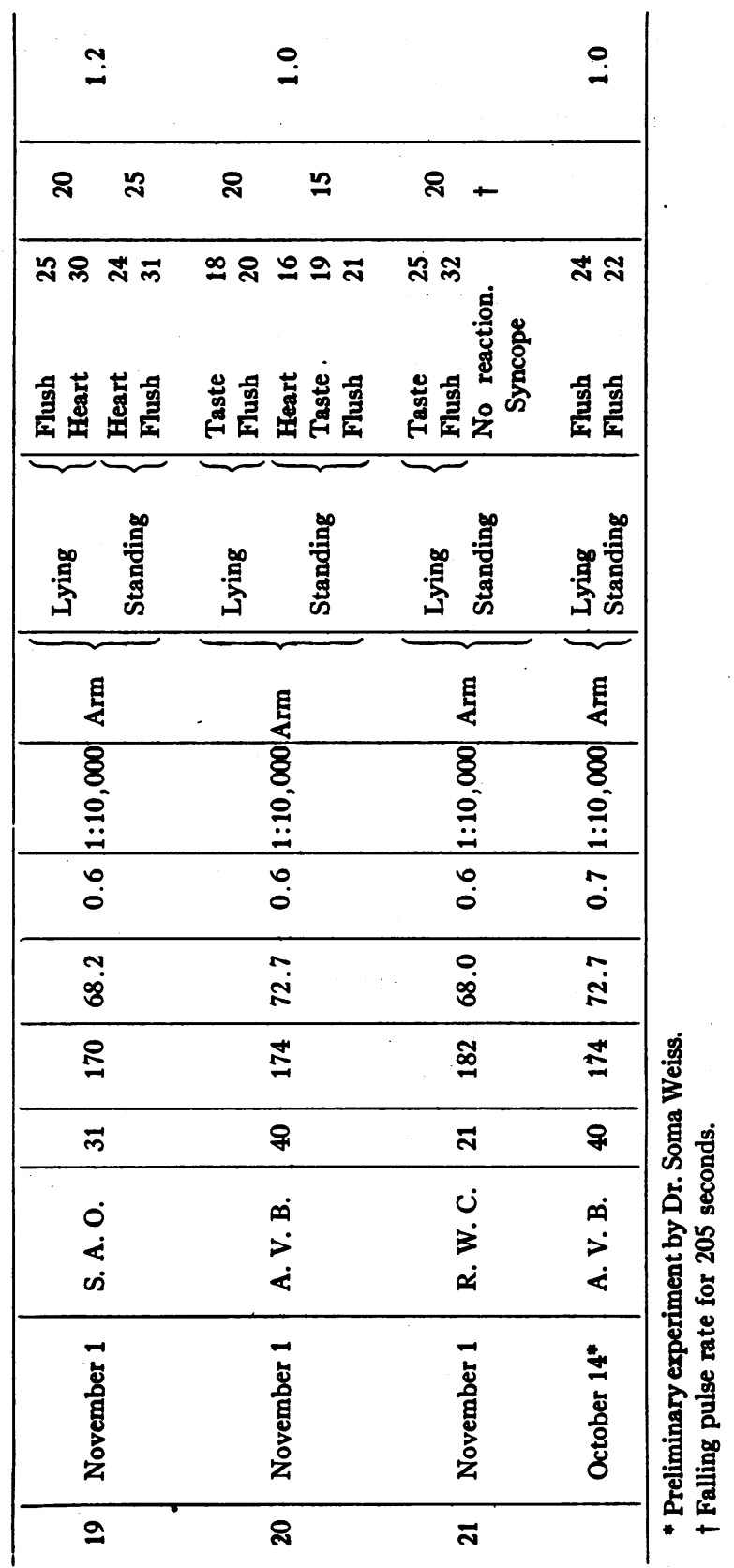


than 10 minutes, unless otherwise noted, before the injection of histamine. In most of the experiments a pulse record was obtained through the use of a cardiotachometer designed by Mr. Paul S. Bauer (3). Histamine phosphate ${ }^{1}$ in varying doses was injected into the internal saphenous vein at the ankle near the internal malleolus of the tibia, or into the ante-cubital vein of the arm. Stop watches were used to register the time of onset of subjective reactions. For the sake of convenience the term "subjective reaction" of histamine is used in contra-distinction to the reaction of the drug upon the heart rate. Although note has been made of various sensations, attention has been

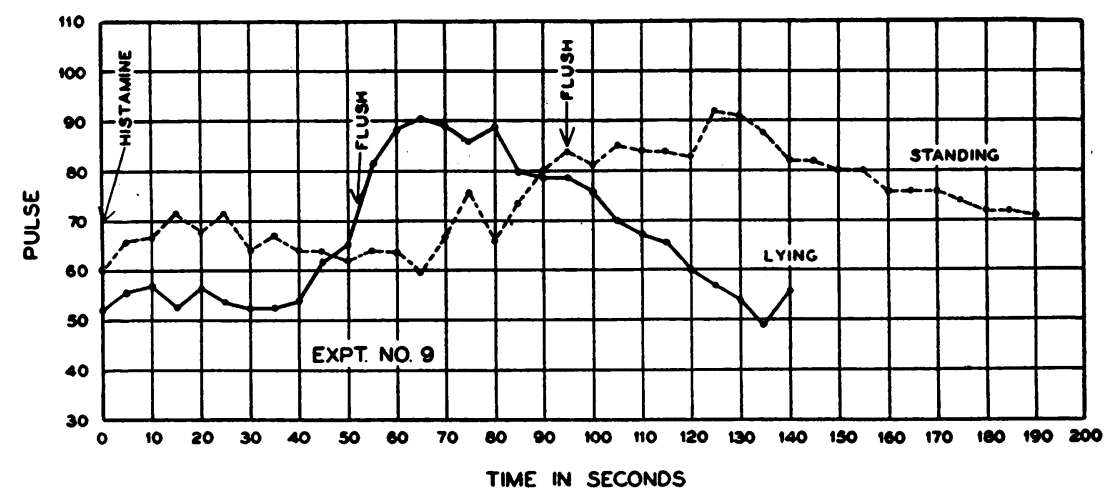

given chiefly to the appearance time of flushing. The data of 21 consecutive experiments are recorded in table 1. A characteristic reaction of the heart rate is illustrated in figure 1.

\section{DISCUSSION}

The data are not sufficient to treat statistically. Upon inspection the first 17 experiments show a longer subjective reaction time in the standing position than was found in the lying position. While standing still the subjective reaction times range from 1.5 to 4.5 times longer than was characteristic when the subjects lay flat. The variations found in the same subject are very likely due to change of physiological

1 The substance used was "Ergamine," Burroughs, Wellcome and Company, and was kindly supplied to us by Dr. Soma Weiss. The required amount of ergamine for a given solution was determined by weight, dissolved in sterile normal salt solution, and used without further sterilization. 
state from time to time, and in different subjects the least differences found may in large part be attributed to states induced by emotional factors. The subjects W. C., P. B., and T. U. were unaccustomed to the experimental procedures instituted in their persons. Emotional influences may have resulted in sufficient vasoconstriction, for example, to hasten the blood flow during standing in these instances.

Evidence that alteration in the blood flow occurred while standing was also noted in the fact that the subjective reactions obtained were in every case less distinct than those occurring in the lying position, and the duration of the effect on the pulse rate, in the cases in which the pulse record was complete, was always longer than in the lying position. Since the pharmacodynamic effect of any substance depends upon its effective concentration, the above findings suggest a greater dilution of histamine in the blood which, under the condition of the experiments, could be brought about only by one or both of two conditions, the accumulation of an increased volume of blood in the legs and abdomen, or a diminished rate of blood flow. The pulse records also in several instances show second waves of increased rate of the heart, suggesting uneven mixing of the histamine in the blood of the legs, with a resulting secondary reaction which may or may not be accompanied by a second flush.

In an effort to produce a more critical reaction point in the standing position by an increase in the concentration of histamine, experiments 12 and 13 were performed. One-tenth cubic centimeter of 1:1000 solution of histamine phosphate was injected instead of the previous dose of $1 \mathrm{cc}$. of 1:10,000 solution. Experiment 12 failed from this point of view owing to syncope of the subject, but it is of interest to note that after standing 5 minutes without a reaction a flush appeared 25 seconds after the subject lay down. In experiment 13 no reaction occurred in 310 seconds. This result suggested that the small volume of the dose of histamine did not find its way into the general circulation. Experiment 14 was undertaken to see if complete washing of the histamine from the small vein into which it was injected would produce a more decisive reaction than occurred in previous experiments. Two cubic centimeters of 1:20,000 solution of histamine were injected. No subjective reaction was experienced in 495 seconds although a moderate increase in pulse rate occurred in 355 seconds. 
The initial and subsequent dilution of the drug was too great to produce an effect in the standing position. In this experiment the reaction time for the lying position was 49 seconds, a short reaction time for the subject A. V. B. This result, in contrast to that of experiment 2. is illustrative of the wide variation possible in the effective concentration of histamine when the subject is in the lying position.

In experiments 15,16 , and 17 the dose of histamine was doubled for the standing posture phase of the experiments without producing a noticeable difference in the reactions. In experiment 16 the early change in pulse rate found during standing may be accounted for by pain produced during repeatedly unsuccessful attempts to complete the venepuncture. It suggests an adrenalin effect upon the heart greater than upon the periphery.

The last phase of this work deals with the velocity of blood flow in the upper part of the body, the injections of histamine being made into the ante-cubital vein with the arm at a right angle to the chest. Experiments 18, 19, 20 and 21 indicate that the velocity of blood flow in both positions is the same, or may be faster in the upright position, presumably on account of the smaller component exposed to the influence of gravity involved in this portion of the circulation. Judging from the results of these experiments as contrasted with the previous ones, it appears probable that blood above the level of the heart may circulate two or three times as fast as blood mounting up from the legs, in the standing position. If this is true it goes far to explain certain apparently paradoxical results obtained by Thompson, Alper and Thompson (4). After injecting brilliant vital red into the cubital vein, the subject standing, these workers found that the time which elapsed before the appearance of the dye in the vein of the foot was longer than that required for the dye to reach the arm after injection into the vein of the foot. It appears possible that several circulations of blood may have occurred above the level of the heart before the dye was sufficiently mixed in the blood to enable it to reach the veins of the foot.

Evidence as to the effect of posture on the velocity of blood flow was also obtained by Thompson, Alper and Thompson (4). They measured the time necessary for a dye, brilliant vital red, to travel from the vein of injection to a distant vein. The subjects were kept in their 
respective positions, lying or standing much longer than was the case in our experiments. Their results indicate that when the dye was injected into a foot vein, the subject standing, the time before the appearance of the dye in the arm vein was at least 1.5 times as long as that required in the prone position. In the paper by Thompson, Thompson, and Dailey (5) it is shown that the mixing time of the dye is greatly prolonged in the standing position over the time required to reach a uniform concentration when the subject lies flat.

Evidence that the output of the heart is reduced in the standing position has previously been published $(6,7)$. The reduction is accompanied by other changes to be observed on change of posture from lying to standing, viz, alterations in blood pressure, pulse rate, respiratory rate, metabolic rate, vital capacity, and alveolar $\mathrm{CO}_{2}$ pressure. Thompson, Thompson and Dailey (5) have published the following account of changes in the blood itself resulting on shifting their subjects from the lying to the standing position:

1. An increase in the number of red cells per cubic millimeter of blood.

2. A corresponding increase in the volume of cells per liter of blood.

3. An increase in the specific gravity of the plasma.

4. An increase in the concentration of plasma protein.

5. A decrease in the concentration of plasma water.

6. A decrease in the total amount of plasma in the blood of the whole body.

These findings are attributed to the loss of protein-free fluid from the blood, presumably due to an increase in capillary pressure in the standing position, occurring chiefly in the lower extremities. The results are difficult to explain except on the basis of greatly reduced blood flow in the standing position. The reader will find in their paper a short review of the evidence concerning the collection of blood in dependent portions of the body, in the standing position.

Richards (8) has attempted an explanation of the shift in volume flow of blood with change of position, based on Poiseuille's law. His conclusion is that constriction of the arterial bed rather than accumulation of blood in dependent parts of the body explains the mechanism involved. Strictly speaking, Poiseuille's law has no applicability to 
the problem of total volume of the blood flow for the following reasons (9):

1. By hypothesis, the law applies only to capillary tubes in which the effect of gravity is not operative. It therefore has no meaning with reference to flow of blood through arteries and veins.

2. The law applies only to true solutions of which blood is not one.

A final answer to the question cannot be given in the light of present knowledge.

It seems necessary to state our recognition of the fact that the total output of the heart and the least time required by the blood to flow between defined points in various parts of the body are two different problems. Nevertheless the correlation between the two is distinct enough to warrant deductions concerning total blood flow from observations on blood velocity. The mass of data on blood velocity obtained by Blumgart and Weiss (2) and by Weiss, Robb and Blumgart (1) in normal subjects and the relation of their normal values to those obtained in hyperthyroidism and in anemia, show about the same correspondence throughout the series as do our published and unpublished data on the minute volume output from the heart in these conditions. We can at least say on good grounds that the diminished velocity of blood flow demonstrated in the standing position by the use of brilliant vital red by Thompson, Alper and Thompson, and by histamine by the present authors is confirmatory of a diminished rate of total blood flow in the standing position. The evidence is strengthened by the occurrence of changes found in the composition of the blood itself, as noted above, and by the factor of dilution of histamine already discussed. It is possible, however, that an increase in the cross-sectional area of the vascular bed, in dependent positions of the body, on standing may account for a decrease in the velocity of blood flow without reducing the volume flow of blood. No mechanism for the maintenance of a normal volume of blood flow under these conditions is known to us.

The facts presented above are all consistent with experimental results, previously published, indicating a reduced output of the heart in the standing position. They are not in accord with the recent data of Grollman (10). This author, measuring the output of the heart with acetylene, has reached the conclusion that the volume out- 
put of the heart is constant in the lying, sitting and standing positions, and that the output of the heart bears a definite relation to the surface area of the body. It is to be remembered that all methods used for the determination of cardiac output in man are indirect. Uncertainty concerning the exact volume flow of blood from the heart must exist because of this fact. Nevertheless, a variable output of blood from the heart on change of position is associated with so many other indications of a change in physiological state as to add significance to such measurements of the cardiac output as we have been able to make. With reference to Grollman's work, we question only the interpretation of his data.

\section{SUMMARY}

Estimations of the least circulation time as measured by reaction to histamine indicate a retardation of the velocity of blood flow in the standing position in man. The authors believe this fact supports previous experimental evidence showing a reduction of the total output of the heart when the subject stands still.

\section{BIBLIOGRAPHY}

1. Weiss, Soma, Robb, George P., and Blumgart, Hermann L., Am. Heart J., 1929, iv, 664. The Velocity of Blood Flow in Health and Disease as Measured by the Effect of Histamine on the Minute Vessels.

2. Blumgart, Hermann L., and Weiss, Soma, J. Clin. Invest., 1927, iv, 15. Studies on the Velocity of Blood Flow. II. The Velocity of Blood Flow in Normal Resting Individuals, and a Critique of the Method Used.

Blumgart, Hermann L., and Weiss, Soma, J. Clin. Invest., 1927, iv, 389. Studies on the Velocity of Blood Flow. VI. The Method of Collecting the Active Deposit of Radium and Its Preparation for Intravenous Injection.

Blumgart, Hermann L., and Weiss, Soma, J. Clin. Invest. 1927, iv, 399. Studies on the Velocity of Blood Flow. VII. The Pulmonary Circulation Time in Normal Resting Individuals.

3. Bauer, Paul S. Description to be published.

4. Thompson, Willard Owen, A'per, Joseph M., and Thompson, Phebe K, J. Clin. Invest., 1928, v, 605. The Effect of Posture upon the Velocity of Blood Flow in Man.

5. Thompson, Willard Owen, Thompson, Phebe K. and Dailey, Mary E., J. Clin. Invest., 1928, v, 573. The Effect of Posture upon the Composition and Volume of the Blood in Man 
6. Field, H., Jr., and Bock, A. V., J. Clin. Invest., 1925, ii, 67. Orthopnoea and the Effect of Posture upon the Rate of Blood Flow.

7. Lawrence, John S., Hurxthal, Lewis M., and Bock, A. V., J. Clin. Invest., 1927, iii, 613. Variations in Blood Flow with Changes in Position in Normal and Pathologic subjects.

8. Richards, Dickinson W., Jr., Proc. Nat. Acad. Sci. 1927, xiii, 354. On the Mechanics of Blood Flow, with Special Reference to the Influence of Change of Posture.

9. Bauer, Paul S. Personal Communication.

10. Grollman, Arthur, Am. J. Physiol., 1929, xc, 210. Physiological Variations in the Cardiac Output of Man. VI. The Value of the Cardiac Output of the Normal Individual in the Basal, Resting Condition. 